\title{
Study of the influence of fiber properties on the inductive method for the characterization of Steel Fiber Reinforced Concrete
}

\author{
Pablo Juan-García, Josep M. Torrents, Rubén D. López-Carreño and Sergio H. Pialarissi Cavalaro
}

\begin{abstract}
The inductive method has shown to be a good method for the estimation of fiber dosage in Steel Fiber Reinforced Concrete. The current formulations reported a strong correlation between inductance and fiber content, as well as orientation. The objective of this article is to study how the inductive measurements are affected by fiber properties, such as its dimensions, magnetic characteristics and the samples temperature. The results, obtained from numerical, simulated and experimental data, propose a shape factor to be applied in the measurements to take into account the aspect ratio of the fibers. As for the temperature, the error introduced is less than a $1 \%$, so a corrective factor is not essential..
\end{abstract}

Index Terms - fiber content, fiber orientation, inductive method, magnetic sensors, nondestructive testing, steel fiber reinforced concrete

\section{INTRODUCTION}

$\mathrm{S}$ teel Fiber Reinforced Concrete (SFRC) has become a widespread material in civil structures, such as tunnels or pavements. The addition of structural fibers to concrete contributes to save time in rebar handling, control cracks and improve the flexural strength of concrete. However, a major factor to ensure the structural safety is the need to control the content and orientation of fibers inside the concrete.

The standard EN 14721 [1] gives two different ways to obtain the dosage of steel fibers in concrete. The first one, suitable for fresh concrete, uses a magnet to separate the fibers from a sample of concrete, previously washed. The second one, for hardened concrete, consists on crushing a sample, from which the fibers are then separated and weighted. Both procedures are time-consuming and environmentally unfriendly, and they do not offer information about the orientation of fibers.

This paragraph of the first footnote will contain the date on which you submitted your paper for review. It will also contain support information, including sponsor and financial support acknowledgment.

This work was supported in part by the Ministry of Science and Innovation through the Research Project BIA2010-17478: Construction processes by means of fibre reinforced concretes and DURADOV (RTC-2015-3617-4).

P. Juan-García and J.M. Torrents are with the Department of Electronic Engineering, Universitat Politècnica de Catalunya, Jordi Girona 1-3, Building C4, $08034 \quad$ Barcelona, Spain (e-mail: pablo.juan@upc.edu; josep.m.torrents@upc.edu)

R.D. López-Carreño and S.H.P. Cavalaro are with the Department of Construction Engineering, Universitat Politècnica de Catalunya, Jordi Girona 1-3, Building C1, 08034 Barcelona, Spain (e-mail: ruben.daniel.lopez@upc.edu; sergio.pialarissi@upc.edu).
Non-destructive techniques (NDT) for the assessment of fiber distribution have been investigated. Different electromagnetic methods have been studied to estimate the content and distribution of fibers in SFRC: based on impedance measurements [2], microwave sensors [3], dielectric measurements [4], X-ray images [5] and many other methods, able to determine the amount of fibers in a certain region of the space. Among them, some studies reported promising results using magnetic sensors for the detection of reinforcing bars [6] and the content of steel fibers [7], due to the high permeability of steel compared to concrete.

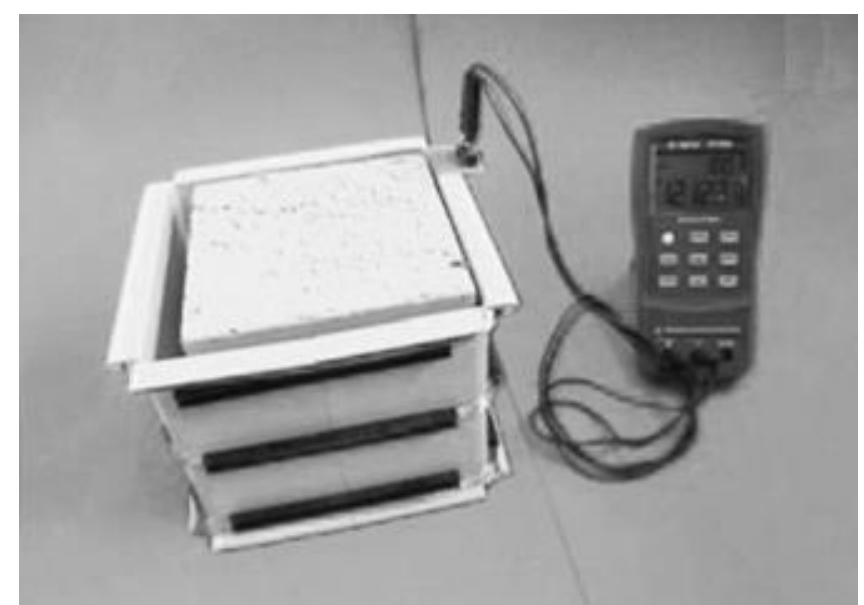

Fig. 1. Inductive method setup.

The inductive method, shown in ¿Error! No se encuentra el origen de la referencia., is an inexpensive procedure that offers relevant information, not only about the fiber dosage of SFRC samples but also about the relative orientation of the fibers [8]. The market offers countless different steel fibers, which leads to different results in the inductive method, mainly caused by its differences in shape and length. Thus, a single calibration curve is needed for each type of fiber [9]. However, it could be sometimes impractical and unaffordable if a wide range of different fibers are tested. Therefore, the study of the fiber properties gives relevant information about the errors introduced by each parameter and the factors that could be applied to reduce the uncertainty of the measurements.

Apart from the shape of the fibers, the chemical composition and manufacturing process of the wide variety of alloys affect the physical properties of steel. Thus, the magnetic properties of the material will modify the inductive response of the 
fibers. The same occurs with the temperature of the samples, as the magnetic permeability is temperature-dependent. The influence of such parameters on the inductive measurements of SFRC remains unstudied.

The objective of this article is to study the influence of different fiber properties in the results obtained with the inductive method. In particular, the relative errors introduced by a change in the aspect ratio of the fibers, the magnetic permeability and the temperature of the samples are studied. Firstly, this work studies the equations that describe the effects of a ferromagnetic needle placed in a constant magnetic field. Then, a corrective factor is proposed and contrasted using numerical simulations. Afterwards, the effects of a change in the fibers temperature are studied with an experimental campaign. In each case, the suitability of a corrective factor is taken into consideration. The application of these corrective factors will improve the accuracy of the inductive method.

\section{PRINCIPLE OF OPERATION}

The inductive method is based on a SFRC sample introduced in the middle of a coil. The high permeability of the steel fibers increases the inductance of the coil, which is measured via an Impedance Analyzer (HP 4192A). Previous works described the method [8], and showed a good correlation between the inductance measurements and the fiber content of the samples tested [10], so this method provides an accurate estimation of the fiber dosage both in cubic and cylindrical concrete samples [11].

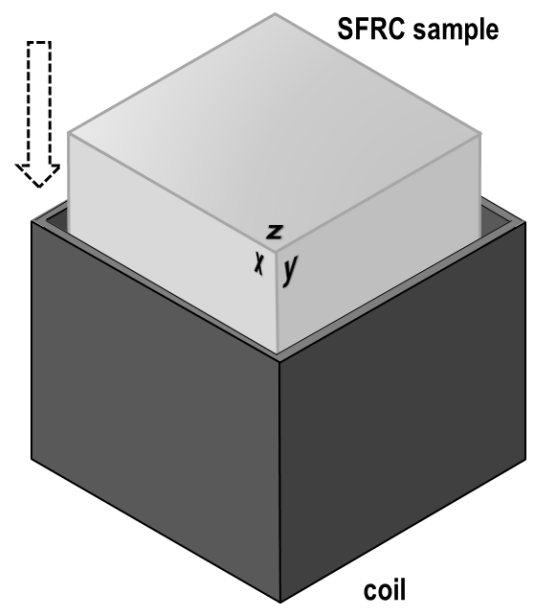

Fig. 2. Measurement procedure

Moreover, the repetition of the measurements along the three main axes $(\mathrm{x}, \mathrm{y}, \mathrm{z})$, gives also relevant information about the orientation of the fibers, as the relative measurements for each of the axis is representative of the amount of fibers that are oriented along that direction. To achieve that, the magnetic field inside the coil must be uniform. Although there are classical solutions to achieve that for regular samples (e.g. solenoid, Helmholtz coils), many works developed different techniques to produce a practically constant magnetic field for any given geometry [12]. Consequently, the inductive method can be not only applied to cubic samples but also to samples of any shape [11].

When the inductive method is performed with cubic samples, the three axes must be defined. As a convention, the z-axis is vertical and parallel to the casting direction of the specimen (see ¡Error! No se encuentra el origen de la referencia.). Then, the axes ' $x$ ' and ' $y$ ' are fixed randomly. Once the inductance of each of the three axes $\left(\Delta \mathrm{L}_{\mathrm{i}}\right)$ is measured, the formulation developed by Cavalaro et al. [10] gives the best estimation for the fiber content determination:

$$
\eta_{i}=1.03 \cdot \sqrt{\frac{\Delta L_{i} \cdot(1+2 \gamma)-\Delta L \cdot \gamma}{\Delta L \cdot(1-\gamma)}}-0.1
$$

where $\gamma$ is a shape factor, that will be discussed in detail in the next section. The dosage of fibers is directly related to the summed inductance for the three axes, $\Delta L$ :

$$
\Delta L=\sum_{i=x, y, z} \Delta L_{i} \quad(2),
$$

As for the estimation of the contribution of fibers for each direction:

$$
C_{i}=\frac{\eta_{i}}{\sum_{i=x, y, z} \eta_{i}} \quad(3),
$$

The measurement of the empty coil is the first measurement needed (reference measurement), as all the equations are referred to the inductance increment. Notice that the same three measurements used for the dosage determination are applied to the orientation estimation.

\section{DEPENDENCE ON ASPECT RATIO OF THE FIBERS}

\section{A. Analytical formulation}

The estimation of the orientation of the fibers lays on the assumption that the fibers are slim enough, so when a fiber is placed perpendicular to the coil axis, its inductance change can be considered negligible, whereas a fiber that is set in the direction of the coil axis, produces a significant inductance change. However, a certain transversal magnetization occurs, so the thickness of the fibers has some influence in the final results.

The shape factor is defined as the relative contribution of the transversal magnetic field with respect to the parallel field. The work of Cavalaro et al. [10] reported that for values of the shape factor $\gamma$ from 0 to 0.05 , the prediction of fiber content in the SFRC samples fitted well with the real orientation of fibers. However, no deeper study has been performed until now for a better estimation of the shape factor. A precise understanding on the real value of this parameter could provide this method with better accuracy, particularly for highly anisotropic scenarios, in which the effects of the aspect ratio of the fibers are much more relevant.

In order to do that, the study of the secondary magnetic field that is created by a single fiber surrounded by a constant magnetic field allows us to define the appropriate corrections for the formulation of the inductive method.

When a magnetically permeable material is immersed in a magnetic field, a magnetization density $(M)$ appears, that is, a magnetization current appears, which results in a secondary magnetic field inside the material. If the external field is weak, steel can be considered a magnetically linear material, which means that

$$
M=\chi_{m} H \quad(4),
$$


where $\chi_{m}$ is the magnetic susceptibility of the material [13]. Therefore, the magnetic field density can be expressed as

$$
B=\mu_{0}(H+M)=\mu H
$$

When an ellipsoid of homogeneous magnetic material is introduced into a uniform parallel field, $B_{0}$, it can be analytically demonstrated that, if the external field is weak enough, the secondary field that appears inside of the ellipsoid will be also uniform, and dependent on the ellipsoid dimensions as well as the magnetic permeability of the material [14]. The magnetic field inside the fiber $\left(B_{1}\right)$ when it is placed on a constant magnetic field $B_{0}$ can be calculated as the addition of its components:

$$
B_{1}=B_{1 x} \boldsymbol{i}+B_{1 y} \boldsymbol{j}+B_{1 z} \boldsymbol{k}(6)
$$

From the point of view of the fiber, the external magnetic field can be decomposed as the two main components of the fiber: parallel $\left(B_{0 \|}\right)$ and perpendicular $\left(B_{0 \perp}\right)$ to the fiber axis. Consequently, for a single fiber with orientation $\alpha$ :

$$
\begin{aligned}
& B_{0 \|}=B_{0} \cdot \cos \alpha \\
& B_{0 \perp}=B_{0} \cdot \sin \alpha
\end{aligned}
$$

The inductance of a single-turn coil can be calculated through the magnetic flux, according to Faraday's law:

$$
\Phi_{m}=\iint_{S} \vec{B} \cdot d \vec{S}
$$

where $\vec{S}$ is, in this context, the section of the fiber that is crossed by the secondary field. Applying equation 9 to the cross section of a single fiber with diameter $\phi$ and length $l$, the parallel and perpendicular fluxes are defined as:

$$
\begin{gathered}
\Phi_{\|}=\frac{\pi}{4} \phi^{2} \cdot B_{1 \|} \\
\Phi_{\perp}=l \phi \cdot B_{1 \perp}(11)
\end{gathered}
$$

In the case of a solenoid, we could define the flux linkage as:

$$
\lambda=N_{f} \cdot \Phi_{m}(12)
$$

where $N_{f}$ represents the number of turns of the solenoid that are crossed by the magnetic field lines of the secondary field. So, finally, if an ideal solenoid is considered, the inductance increment of the sensing coil for a single fiber can be approximated as:

$$
\Delta L \approx N_{f} \cdot\left(\Phi_{\|} \cdot \cos \alpha+\Phi_{\perp} \cdot \sin \alpha\right)(13)
$$

As for the determination of the secondary field, each single fiber can be modelled as a prolate ellipsoid, in which the semimajor axis (a) is much longer than the two semi-minor axes (b). Thus, for the calculations, the semi-major axis $(a)$ of the ellipsoid is defined as

$$
a=\frac{l}{2} \quad(14)
$$

The derivation of the expressions for $B_{1}$ is described in detail in [15]. The expressions for the parallel and perpendicular components of $B_{1}$ are calculated as follows:

$$
\begin{aligned}
& B_{1 \|}=\frac{\mu_{i}}{\mu_{e}} \frac{B_{0 \|}}{1+\left(\frac{a b^{2}}{2}\right)\left(\frac{\mu_{i}}{\mu_{e}}-1\right) E_{1}(0)} \\
& B_{1 \perp}=\frac{\mu_{i}}{\mu_{e}} \frac{B_{0 \perp}}{1+\left(\frac{a b^{2}}{2}\right)\left(\frac{\mu_{i}}{\mu_{e}}-1\right) E_{2}(0)}
\end{aligned}
$$

where $\mu_{i}$ and $\mu_{e}$ are the magnetic permeability inside and outside the fiber, and

$$
\begin{aligned}
& E_{1}(0)=\int_{0}^{\infty} \frac{d s}{\left(s+a^{2}\right) R_{s}} \\
& E_{2}(0)=\int_{0}^{\infty} \frac{d s}{\left(s+b^{2}\right) R_{s}}
\end{aligned}
$$

and also

$$
R_{s}=\sqrt{\left(s+a^{2}\right)\left(s+b^{2}\right)^{2}}
$$

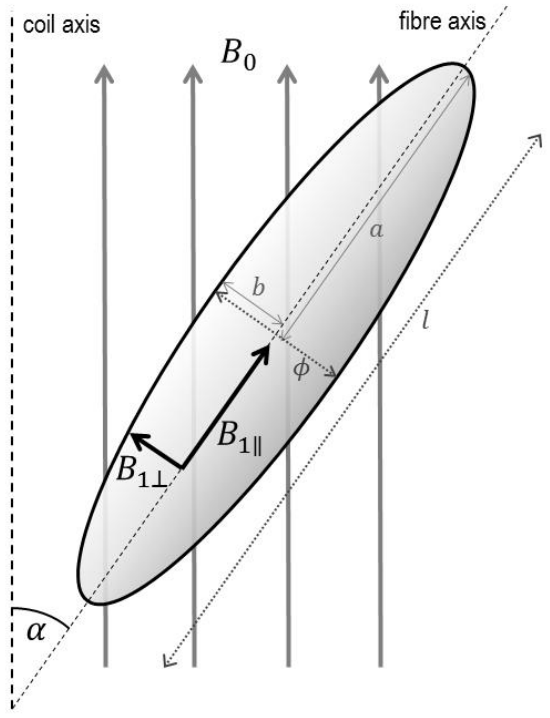

Fig. 1. Magnetic ellipsoid with an arbitrary inclination in a constant magnetic field.

\section{B. Simulations of the model}

In order to evaluate the accuracy of the formulation, a single rod was simulated using COMSOL Multiphysics v4.3. A magnetic cylinder (length of $10 \mathrm{~cm}$ and $1 \mathrm{~cm}$ of diameter) was placed in the middle of a solenoid, which simulates a fairly constant magnetic field. The rod, initially aligned with the axis of the solenoid, was rotated in steps of 15 degrees, so in the end (90 degrees), it was placed perpendicular to the solenoid axis. The inductance of the solenoid was registered for each angle.

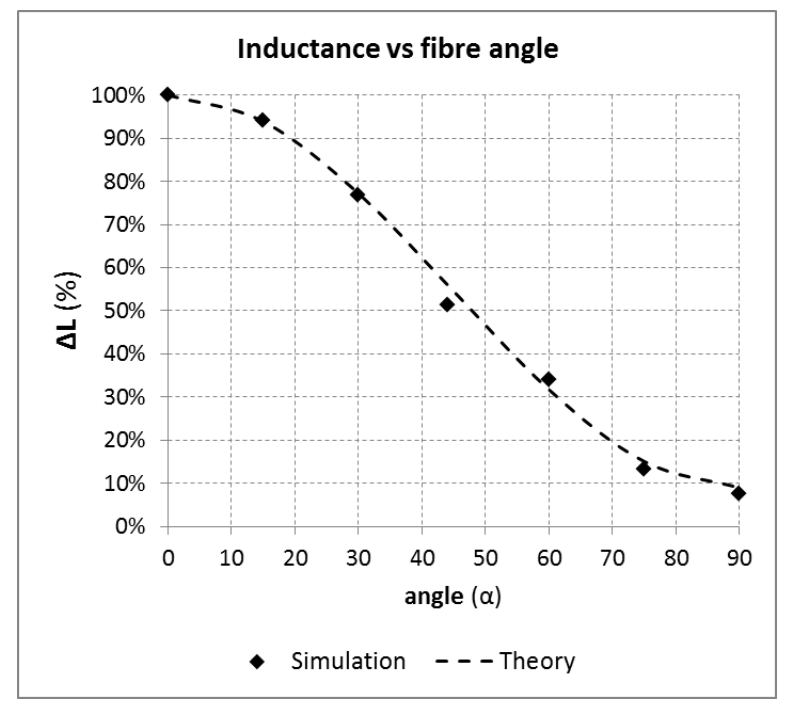

Fig. 2. Inductance increase of a steel rod rotated in the middle of a solenoid.

The simulations are compared with the analytical model, by means of applying equation 13 to evaluate the inductance increment caused by the steel rod, using the expressions that define the parallel and transversal magnetic field described in equations 15-19. Fig. 2 shows the good agreement between the 
simulation results and the theoretical values for an ellipsoid with the dimensions given for the rod $\left(\mu_{i}=100\right)$.

\section{Shape factor determination}

With the aim to include the aspect ratio of the fibers in the inductance measurements, the formulation proposed by Cavalaro et al. [10] defined the shape factor, that can be expressed as the ratio between the transversal and the longitudinal inductance contribution.

$$
\gamma=\frac{\Delta L_{\alpha=90} \mathrm{o}}{\Delta L_{\alpha=0}}
$$

The calculation of the parallel and perpendicular flux obtained from the ellipsoid modelling gives a precise estimation of the shape factor, which can be introduced in the formulation of the inductive method to improve its accuracy.

The first step in the calculation of the shape factor consists on the determination of the magnetic permeability of the fibers. In practice, it is often difficult to precisely know the permeability of the fibers of different manufacturers, as there are countless alloys in the market, which result in lots of different values of permeability. A simple procedure to achieve that is to wrap a wire around one of the fibers and measure the inductance of the solenoid with and without the steel core (Fig. 3). The relation between both measurements gives the relative permeability of the material.

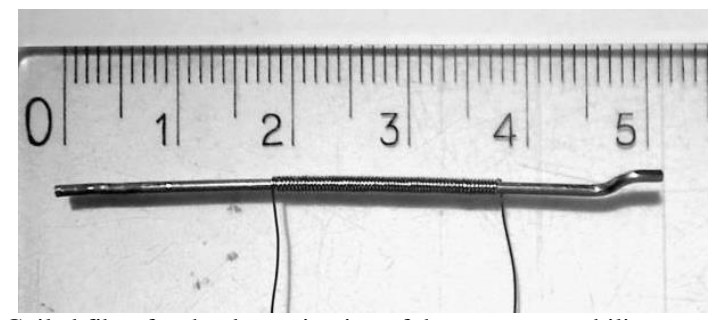

Fig. 3. Coiled fiber for the determination of the core permeability

For the determination of the permeability, a single fiber was used (Dramix Twinplate, diameter: $1 \mathrm{~mm}$, length: $50 \mathrm{~mm}$ ). A 64-turn solenoid, coiled using a $0.24 \mathrm{~mm}$ wire, had an inductance of $0.34 \mu \mathrm{H}$, that was increased up to $12.25 \mu \mathrm{H}$ when the fiber was placed inside it. Then, a relative permeability of 36 is derived at a frequency of $1 \mathrm{kHz}$.

Fig. 4 shows the value of the shape factor for different aspect ratios, in the range of dimensions of the most widely used fibers, considering a relative permeability $\mu_{r}=36$, as it has been calculated before. To obtain the different curves, the shape factor is obtained from equation 20, applying equation 13 for the calculation of the transversal and longitudinal field. Then, for each geometry, a different value of $\gamma$ is obtained, and this will properly modify the calibration curve for each type of fiber.

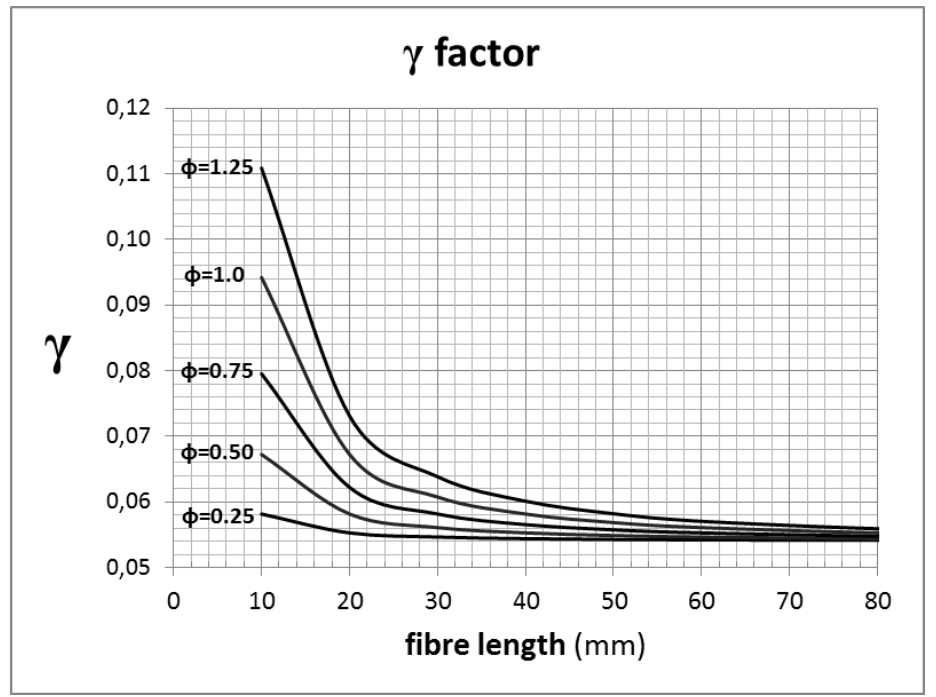

Fig. 4. Analytical calculation of the shape factor as a function of fiber dimensions $\left(\mu_{\mathrm{r}}=36\right)$.

\section{Simulations}

The calculation of the shape factor is particularly relevant for scenarios in which the fibers are oriented with certain anisotropy, since for homogeneous distributions the effects of the aspect ratio are compensated by the randomness of the sample. Therefore, the formulation was validated by simulations of highly anisotropic situations. The simulation, performed with the same parameters than the previous section, consisted on eight cylinders, arranged to set two different scenarios: the first one consisted on the eight cylinders set in the same direction, and the other one, with the fibers radially distributed in a plane (Fig. 5).
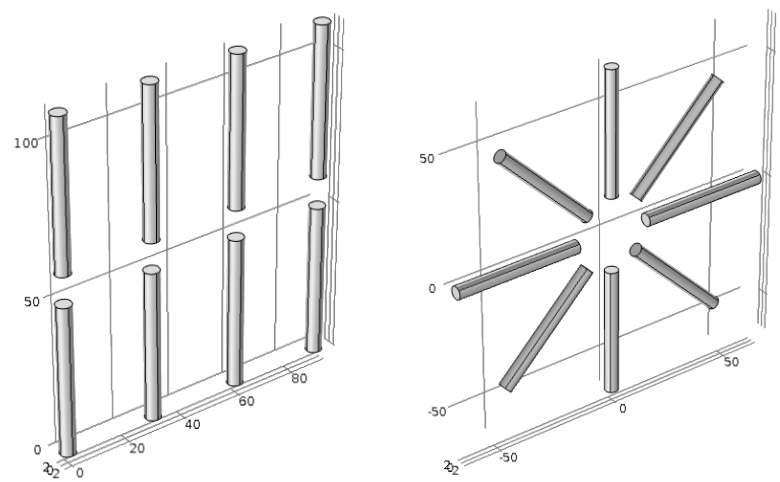

Fig. 5. Simulation distribution for single-axis contribution (left) and doubleaxis contribution (right)

\section{TABLE I}

UNITS FOR MAGNETIC PROPERTIES

shows the results of the simulations for the two abovementioned scenarios. Two different evaluations were performed: the first considers the equations proposed by Cavalaro et al [10] without shape factor correction, that is $\gamma=$ 0 . The second one, considers the same formulation with the inclusion of the shape factor that results from the curves shown in the previous section. It can be observed that in the 
case of highly anisotropic samples, a proper selection of the $\gamma$ factor leads to an error reduction around a $25 \%$ in the case of a single-axis contribution or a $5 \%$ in the double-axis contribution.

TABLE I

UNITS FOR MAGNETIC PROPERTIES EXPECTED VS SIMULATED RESULTS

\begin{tabular}{ccccc}
\hline \hline & Expected & \multicolumn{2}{c}{ Simulated } \\
\hline \multirow{5}{*}{ Single-axis } & $\mathrm{x}$ & $100 \%$ & $\begin{array}{c}\text { Without correction } \\
(\gamma=0)\end{array}$ & $\begin{array}{c}\text { With correction } \\
(\gamma=0.09)\end{array}$ \\
\cline { 3 - 5 } & & & $67.4 \%$ & $91.6 \%$ \\
& $\mathrm{y}$ & $0 \%$ & $14.0 \%$ & $0.0 \%$ \\
& $\mathrm{z}$ & $0 \%$ & $18.7 \%$ & $8.4 \%$ \\
& & & & $47.4 \%$ \\
& $\mathrm{x}$ & $50 \%$ & $42.0 \%$ & $47.4 \%$ \\
& $\mathrm{y}$ & $50 \%$ & $42.0 \%$ & $5.1 \%$ \\
\hline \hline
\end{tabular}

\section{DEPENDENCE ON STEEL COMPOSITION}

Apart from geometrical considerations, the composition of the fibers also affects the error of the measurement. Specifically, as a consequence of the different chemical compositions of steel, the fibers could present a different magnetic permeability from one to another. Consequently, the above presented results would change for fibers with different permeability, so a study on the influence of the variation of permeability values is performed.

Using the formulation for ellipsoidal fibers, equations 15-16 could be formulated in terms of the demagnetization factor $N_{x}$,

$$
N_{x}=\left(\frac{a b^{2}}{2}\right) E_{x}(0) \quad(21)
$$

Then, considering a paramagnetic surrounding medium $\left(\mu_{e}=\right.$ 1),

$$
\begin{aligned}
& B_{1 \|}=\frac{\mu_{i} \cdot B_{0 \|}}{1+N_{\|}\left(\mu_{i}-1\right)}(22) \\
& B_{1 \perp}=\frac{\mu_{i} \cdot B_{0 \perp}}{1+N_{\perp}\left(\mu_{i}-1\right)}(23)
\end{aligned}
$$

In the case of prolate ellipsoids (e.g.: needles), one can consider $N_{\perp} \approx \frac{1}{2}$ and $N_{\|} \approx 0$. Then, the shape factor for fibers with high aspect ratio $\left(A_{r}=l /_{\phi}\right)$ could be approximated as:

$$
\gamma \approx \frac{2}{\mu_{i}+1}
$$

Therefore, Fig. 6 shows that a change in the permeability entails a large variation in the calculation of the shape factor. Consequently, the steel composition and manufacturing process is relevant in the formulation presented before. It should be clarified that the relative permeability of steels usually ranges from 100 to more than 250. However, Bowler [16], reported that in the case of quenched steel, the magnetic permeability dramatically decreased to figures around 30 at 1 $\mathrm{kHz}$, in agreement with the values measured in the previous section.

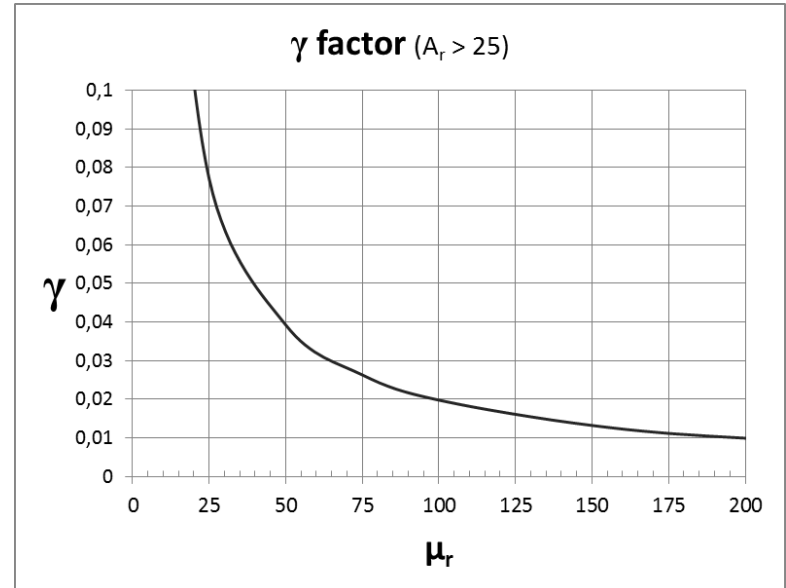

Fig. 6. Effect of magnetic permeability on the shape factor for fibers with high aspect ratio

\section{DEPENDENCE ON THE TEMPERATURE OF THE SAMPLES}

The third aspect studied was the influence of a variation in the temperature of the samples on the inductive measurements. Although in laboratory conditions, the inductive method should be performed at room temperature, it is not always possible to do so in field measurements.

According to the Curie-Weiss law, the magnetic susceptibility of a material increases as the temperature is increased, until it reaches the Curie Temperature, which is far higher than atmospheric temperatures for common steel alloys. Then, a change in the inductance could be expected if the temperature changes. For a small range of temperatures, the susceptibility increment could be considered almost linear [17]. Thus, a corrective factor could be introduced in the formulation if the temperature had a determinant influence in the measurements. However, the thermal factor must be different for each kind of fiber, so it would require its own calibration curve.

For the temperature dependence measurements, 22 cubic samples were casted (15 cm side), 10 of them produced with conventional concrete, and 10 with self-compacting concrete. Three different fiber dosages were tested, namely 30, 45 and $60 \mathrm{~kg} / \mathrm{m}^{3}$. The fibers were BASF Masterfiber 502, with the same dimensions than in the previous section. Once the samples were set, they were placed in a climate-controlled room, with a constant humidity of $80 \%$ and when they reached the desired temperature, they were measured individually.

To evaluate the dependence on the temperature of the inductive measurements, the three main axes of each sample were measured, and the sum of the three of them was studied. The measurements performed at 0 degrees were taken as a reference, and the same samples were measured at 10, 20 and 30 degrees. So the inductance at each temperature is measured with reference to the measurement at 0 degrees, while the equipment is kept at room temperature. Then,

$$
\Delta L_{20^{\circ}}=\Delta L\left(T=20^{\circ}\right)-\Delta L\left(T=0^{\circ} \text { o }\right)(25)
$$




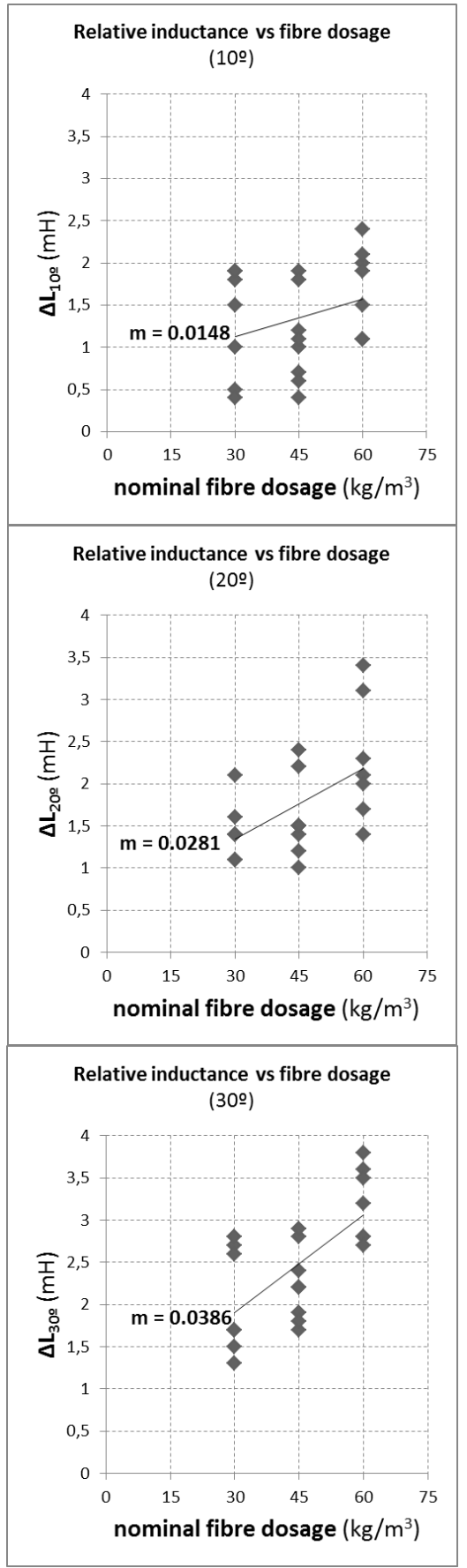

Fig. 7. Inductance increment for three different temperature of the samples

Fig. 7 shows that the measurements are dependent both on the content of fibers and the temperature, as it was expected, since the changes in the permeability due to the temperature increase of each fiber leads to a proportional change in the inductance of the whole sample. Therefore, the results will be normalized by the value of the inductance at $0{ }^{\circ} \mathrm{C}$, which is highly correlated with the fiber content, obtaining a relative value that will give information about the uncertainty related to a change in the temperature.

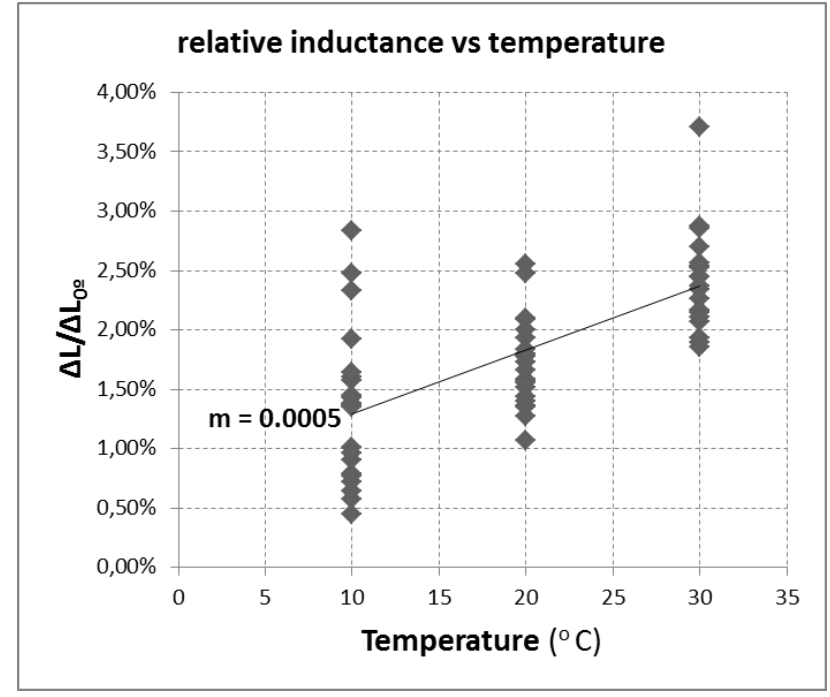

Fig. 8. Relation between the inductance of the samples and the temperature (referred to $0^{\circ} \mathrm{C}$ )

Fig. 8 shows the relative increment of the inductance as the temperature changes. According to those results, the measurements show an error of a $0.05 \% /{ }^{\circ} \mathrm{C}$. That is, if the measurements are performed in normal field conditions (between 10 and $30{ }^{\circ} \mathrm{C}$ ) the error introduced by the temperature is smaller than $1 \%$, which is under the typical uncertainties of the method [18]. Therefore, it is not essential to introduce a correction factor to counteract the temperature effects, which would be impractical to perform if different fiber types are used.

\section{CONCLUSIONS}

The inductive method gives useful information about the distribution and orientation of fibers in Steel Fiber Reinforced Concrete, as it was reported in previous works. This article reported how many properties of the fiber could influence the final result, namely the aspect ratio, the magnetic permeability and the temperature. Moreover, the possibility of introducing a corrective factor for each of them was taken into consideration.

The aspect ratio of the fibers plays a secondary role when the fibers are randomly distributed. However, in some situations in which there is a preferential orientation, the results get significantly affected by the diameter of the fibers (particularly for fibers with smaller aspect ratios). In order to deal with the error introduced by the aspect ratio of the fibers, a shape factor was proposed and evaluated with simulations and measurements. The curves to select the adequate shape factor for the most common steel fibers have been presented. The simulations and measurements showed that in cases of high anisotropy, there is an error reduction of almost a $25 \%$ by the correct choice of the shape factor.

Regarding the permeability of the fibers, if we consider the range of permeabilities of quenched steels, the results derived from the ellipsoidal model of the fibers showed that the error introduced by a change in the magnetic permeability of the fibers is highly relevant. The effects of the permeability of the 
fibers must be corrected either by measuring it on an individual fiber (which is sometimes impractical) or through a calibration curve performed for each fiber type.

Finally, as for the temperature of the fibers, it was demonstrated experimentally that an increase in the temperature leads to a proportional increase in the inductance of the coil. Thus, a thermal factor could be applied in the measurements, if a calibration curve is obtained for each specific type of fiber. Nonetheless, if the temperature of the environment is between 10 and 30 degrees, the error would be less than a $1 \%$, which is acceptable, since it is comparable to the typical uncertainties associated to the measurement setup.

\section{REFERENCES}

[1] EN-14721, "Test method for metallic fiber concrete. Measuring the fiber content in fresh and hardened concrete," vol. BS EN 14721:2005+A1:2007, ed, 2005.

[2] J. M. Torrents, et al., "Electrical impedance spectroscopy as a technique for the surveillance of civil engineering structures: considerations on the galvanic insulation of samples," Measurement Science \& Technology, vol. 18, pp. 1958-1962, Jul 2007.

[3] S. Van Damme, et al., "Nondestructive determination of the steel fiber content in concrete slabs with an open-ended coaxial probe," IEEE Transactions on Geoscience and Remote Sensing, vol. 42, pp. 2511-2521, Nov 2004.

[4] J. Karlovsek, et al., "Frequency-dependant dielectric parameters of steel fiber reinforced concrete," in 14th International Conference on Ground Penetrating Radar (GPR), 2012, pp. 510-516.

[5] C. Redon, et al., "Assessment of fiber orientation in reinforced concrete using Fourier image transform," Journal of Microscopy, vol. 191, pp. 258-265, 1998.

[6] P. Gaydecki, et al., "A portable inductive scanning system for imaging steel-reinforcing bars embedded within concrete," Sensors and Actuators A: Physical, vol. 84, pp. 25-32, 2000.
M. Faifer, et al., "Nondestructive Testing of Steel-FiberReinforced Concrete Using a Magnetic Approach," IEEE Transactions on Instrumentation and Measurement, vol. 60, pp. 1709-1717, 2011.

[8] J.M. Torrents, et al., "Inductive method for assessing the amount and orientation of steel fibers in concrete," Materials and Structures, vol. 45, pp. 1577-1592, 2012/10/01 2012.

[9] H.-J. Wichmann, et al., "Ein praxisgerechtes Messverfahren zur Bestimmung der Fasermenge und -orientierung im Stahlfaserbeton," Beton- und Stahlbetonbau, vol. 108, pp. 822-834, 2013.

[10] S. H. P. Cavalaro, et al., "Improved assessment of fiber content and orientation with inductive method in SFRC," Materials and Structures, pp. 1-15, 2014/03/11 2014.

[11] S. H. P. Cavalaro, et al., "Assessment of fiber content and 3D profile in cylindrical SFRC specimens," Materials and Structures, pp. 1-19, 2015/01/09 2015.

[12] J. M. Torrents, et al., "Method for forming a magnetic field of arbitrary shape from an established template," presented at the 19th Symposium IMEKO TC4 Symposium and 17th IWADC Workshop, Barcelona, Spain, 2013.

[13] H. A. Haus and J. R. Melcher, Electromagnetic Fields and Energy. NJ: Massachusetts Institute of Technology OpenCourseWare, 1989.

[14] J. A. Stratton, Electromagnetic Theory. New York \& London: McGraw-Hill Book Company, 1941.

[15] A. A. Kaufman, et al., Principles of the Magnetic Methods in Geophysics vol. 42. Amsterdam [etc]: Elsevier, 2008.

[16] N. Bowler, "Frequency-Dependence of Relative Permeability in Steel," AIP Conference Proceedings, vol. 820, pp. 1269-1276, 2006.

[17] K. H. J. Buschow and F. R. de Boer, Physics of Magnetism and Magnetic Materials. New York [etc.]: Kluwer Academic Publishers, 2004.

[18] S. H. P. Cavalaro, et al., "Influence of temperature on the assessment of fiber content and orientation with the inductive method," presented at the 7th International Conference Fiber Concrete, Prague, Czech Republic, 2013. 